\title{
Effect of Solvent on the Phytochemical Extraction and GC-MS Analysis of Gymnema sylvestre
}

\author{
Sundarapandian Subramanian', Mohammed Junaid Hussain Dowlath', Sathish Kumar Karuppannan ${ }^{1}$, \\ Saravanan $\mathbf{M}^{2}$, Kantha Devi Arunachalam ${ }^{1, *}$
}

Sundarapandian Subramanian', Mohammed Junaid Hussain Dowlath', Sathish Kumar Karuppannan', Saravanan $\mathbf{M}^{2}$, Kantha Devi Arunachalam ${ }^{1, *}$

${ }^{1}$ Center for Environmental Nuclear Research, Directorate of Research, SRM Institute of Science and Technology, Kattankulathur, Chennai 603203, INDIA. ${ }^{2}$ Department of Biotechnology, School of Bioengineering, SRM Institute of Science and Technology, Kattankulathur, Chennai 603203, INDIA.

\section{Correspondence}

\section{Kantha Devi Arunachalam}

Center for Environmental Nuclear Research, Directorate of Research, SRM Institute of

Science and Technology, Kattankulathur,

Chennai 603203, INDIA

E-mail: kanthad.arunachalam@gmail.com History

- Submission Date: 10-04-2020;

- Review completed: 27-04-2020;

- Accepted Date: 08-05-2020;

DOI : 10.5530/pj.2020.12.108

Article Available online

http://www.phcogj.com/v12/i4

Copyright

(C) 2020 Phcogj.Com. This is an open access article distributed under the terms of the Creative Commons Attribution 4.0 International license.

\section{ABSTRACT}

The medicinal plant Gymnema sylvestre found in the Indian subcontinent and Srilanka is known for its anti-diabetic, diuretic, anti-obesity, anti-cancer, antimicrobial, anti-inflammatory properties. The current study is focused on the phyto compound extraction efficiency of different solvents like ethanol, methanol, ethyl acetate, hexane, benzene and chloroform by gas chromatography-mass spectrometry analysis of Gymenma sylvestre. From the results, it is concluded that $G$. sylvestre leaves extracts contains more than 38 phyto compounds with natural antioxidants potential. Further analysis of the extract will help in identifying the effective compounds which can be of potent use in the pharmacological field.

Key words: Gymnema sylvestre, Medicinal plants, chromatography, plant constituents, Cold maceration.

\section{INTRODUCTION}

Since time immemorial, various parts of the plants such as leaves, roots, stem etc. are being used to treat number of diseases and infections. India is blessed with and is a source for variety of herbal plants with medicinal properties. Gymnema sylvestre is a woody climber shrub from the family of Apocynaceae. Gymnema genus has 50 species in the genus. It is native to India, Australia, Africa and China. It is found to be grown well in the tropical regions. Gmnema sylvestre is an important plant with medicinal properties. In local languages of India, it is called as Sakkarai kolli which literally means "destroyer of sugar"1. Because of the medicinal importance, this plant is used in the preparation of formulated medicine for treating various health ailments. In Indian Ayurvedic medicine system, G.sylvestre is used for treating diabetes. Therapeutically, crude extract of this plant is used as a diuretic, to cure stomachic, eye complaints, asthma, chronic cough, cardiopathy, constipation, piles etc. Apart from these, various pharmacological and biological activities such as antibacterial, antiviral, antifungal, antiinflammatory, anticancer has been reported ${ }^{2-5}$.

Due to the side effects associated with allopathic medicines, in recent years, research interests have turned towards plant-based phytochemicals in treating various diseases ${ }^{6}$. Phytocompounds from medicinal plants are used in formulations of various healthcare nutraceuticals and cosmetics products. The phytocompounds of G. sylvestre has been effective in controlling diabetes ${ }^{7}$. The phytochemical compounds like phenols, flavonoids, terpenoids, saponins, tannins of the plants are the base for modern day allopathic medicines. The active components of allopathic drugs constitute about 25 $-40 \%$ of plant-based origins ${ }^{8}$. The literature survey revealed that no work has been done to compare the effect of solvents on biochemical constituents of G.sylvestre plant extracts. To the best of our knowledge, no study has been conducted to study the effect of agroclimatic location on the antioxidant activity of $G$. sylvestre leaves. In this study, the plant samples collected from different regions of Tamil Nadu were evaluated for their antioxidant activities and the sample showing high level of antioxidant activity was extracted with six different solvents like ethyl acetate, ethanol, methanol, chloroform, hexane and benzene. The phytochemical constituents of the crude extracts of G. sylvestre of the different solvents were characterized by GCMS analysis. This study will reveal the relationship between the effect of sampling locations to the quantity and quality of the phytocompounds and its antioxidant activities of the plant sample and also the effect of solvents on the phytochemical availability in the crude extract. This will help to select the suitable solvent based on the actual application of the extracts.

\section{MATERIAL AND METHODS}

\section{Chemicals}

All the chemical used in the study were of analytical grade and are purchased from Sisco Research Laboratories, India. The DPPH was purchased from Sigma, India.

\section{Plant sample}

G. sylvestre leaves collected during the month of November 2016. The collected plant samples were identified and authenticated by Dr. G.V.S Murthy, Botanical Survey of India (Ref No: BSI/ SRC/5/23/2016/Tech/215). The leaves were washed with running tap water, distilled water and shade dried at room temperature. The dried leaves were ground by using a laboratory blender. The pulverized samples were stored in cold storage for further usage. 
For the present study, during the same month, the G. sylvestre plants were collected from seven different locations of Tamil Nadu (Figures $1 \& 2$ ) such as

1. Shenbagadevi falls at Coutrallam on the Western Ghats in Tirunelveli District

2. Thirunel, Kottamalai at Padavedu, Thiruvannamalai District

3. Irulars Tribal Women's Welfare Society at Chengalpattu District
4. Muniyankudisai Village at Arni, Tiruvanamalai District

5. Velliangiri Hills at the Western Ghats border of Coimbatore District

6. Anthyodhaya sangham at Trichy

7. Gandhi gram Trust at Dindigul district

Effect of sampling location on antioxidant activity

The effect of locations on antioxidant activity of G.sylvestre plants was estimated by DPPH method following the method $^{9}$ of Blois, 1958. The

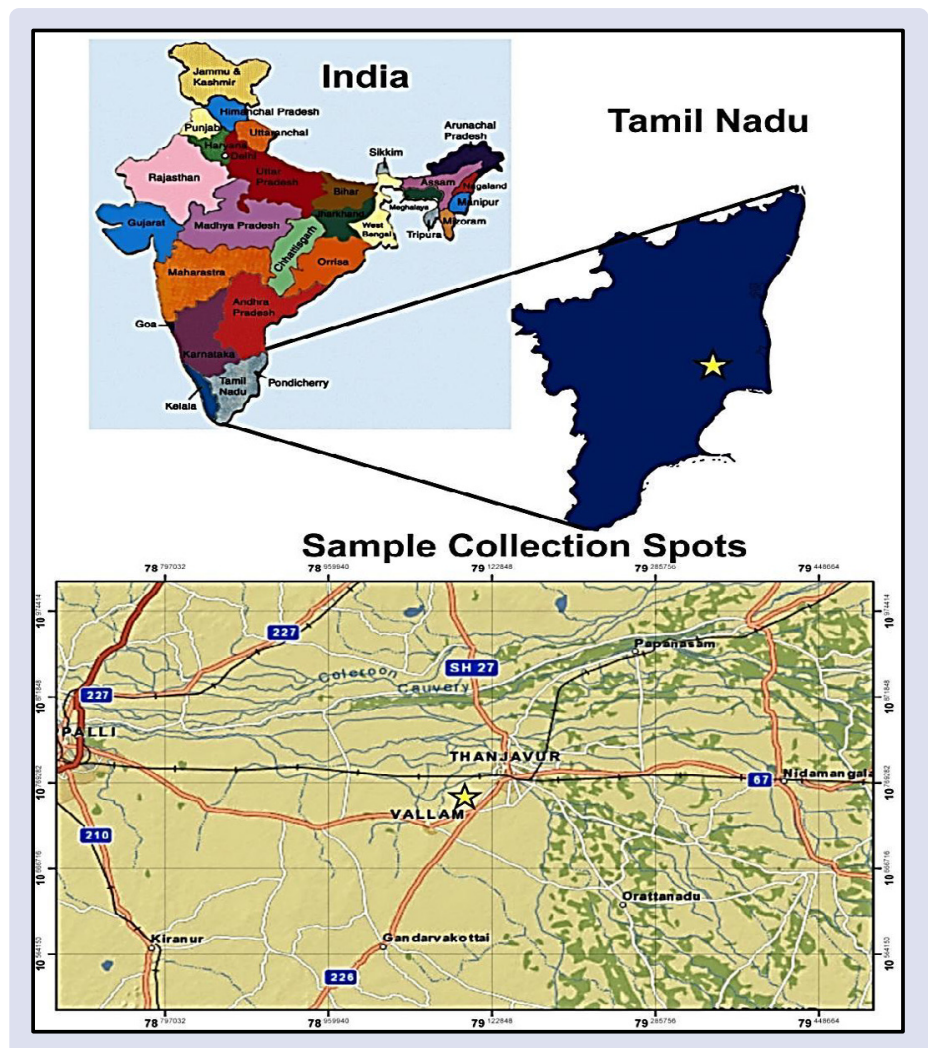

Figure 1: Shows the G.sylvestre collection spot (at column width).

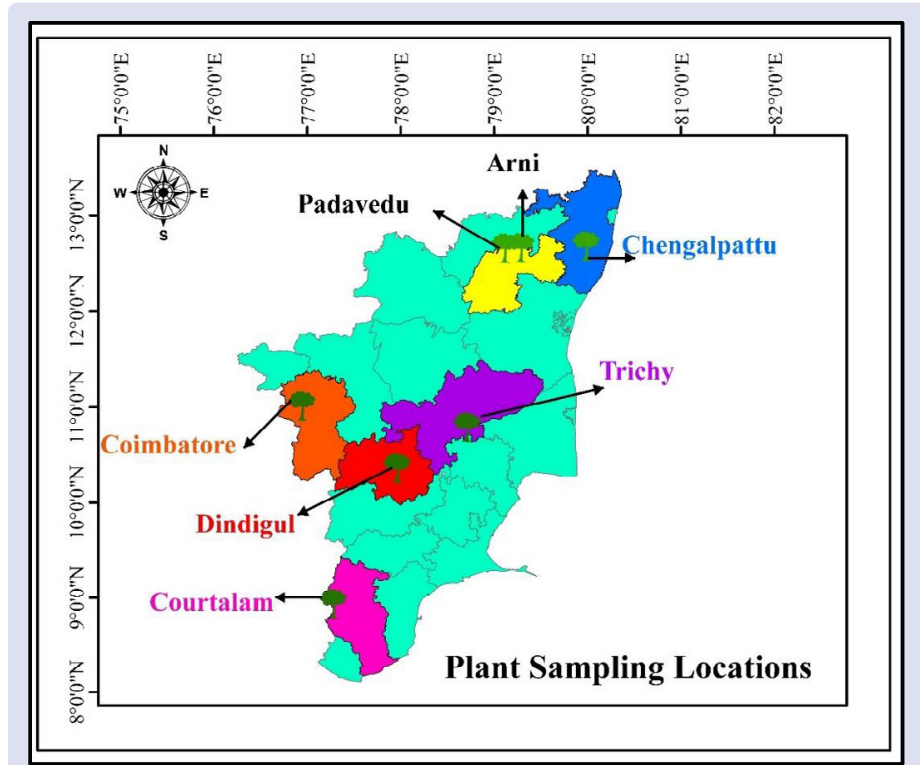

Figure 2: Gymnema sylvestre collection spots around Tamil Nadu (at column width). 
reaction mixture $(0.1 \mathrm{mM}$ DPPH and extract) was vortexed, incubated and its absorbance was measured at $517 \mathrm{~nm}$. The scavenging ability of the plant extracts was calculated using the following equation (1)

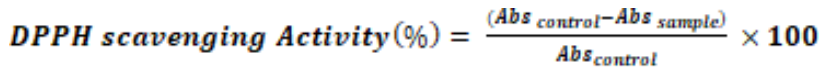

Where, $\mathrm{Abs}_{\text {control }}$ is the absorbance of DPPH without sample; $\mathrm{Abs}_{\text {sample }}$ is the absorbance of DPPH with sample ${ }^{10}$ Cieśla et al.

\section{Extraction procedure}

The plant sample obtained from Coutrallam is used for extraction. G.sylvestre leaf powder $50 \mathrm{~g}$ was extracted with $500 \mathrm{ml}$ ethanol by cold maceration method for $72 \mathrm{~h}$. After extraction, it was filtered using Whatman N0. 41 filter paper to obtain solid particle free extract and the solvent was evaporated to dryness under vacuum using a rotary evaporator. The crude extract obtained was stored at $4{ }^{\circ} \mathrm{C}$ for further usage. The same procedure was followed for all other solvents like methanol, benzene, hexane, ethyl acetate and chloroform.

\section{Estimation of chemical constituents by GC-MS}

To determine the various volatile bioactive compounds present in each solvent extract, GC-MS analysis was conducted using SHIMADZU, QP2010 PLUS following the injecting temperature at $250^{\circ} \mathrm{C}$, column temperature at $50^{\circ} \mathrm{C}$, pressure at $29.7 \mathrm{kPa}$ and column flow rate at 0.72 $\mathrm{ml} / \mathrm{min}$. The total running time for the sample was 50 minutes. Based on the retention time the phytochemical compounds in various solvent extracts were identified by matching MS with available standards using NIST and Willey library.

\section{RESULTS}

\section{DPPH radical scavenging activity}

The influence of source of plant collection on the antioxidant activity was studied by DPPH method. The antioxidant activity $\%$ is presented in the Table 1. Significant influence of the location on antioxidant activity was found. Variation in the activity was witnessed with highest activity observed in the plants collected from Shenbagadevi falls at Coutrallam on the Western Ghats in Tirunelveli District with $73.40 \%$ followed by Anthyodhaya sangham at Trichy $66.10 \%$. The plants collected from Gandhigram Trust at Dindigul district showed the least activity of $36.70 \%$.

\section{Chemical constituents of various extracts}

The leaves obtained from coutrallam is used for the extraction and identification of phytochemicals using different solvents such as hexane, benzene, chloroform, ethyl acetate, methanol and ethanol. The results pertaining to GC-MS analysis (Figure 3) of the hexane crude extract of G.sylvestre leaves was analysed using GC-MS which lead to the identification of 36 different organic compounds is listed in the Table 2 with Phytol (10.294\%), Squalene (10.282\%), Tetratriacontane (>14 $\%)$ at various time intervals, n-Hexadecanoic acid (5.186 \%), Eicosane (>10 \%) at various time intervals, Stigmasterol (2.484 \%), Phthalic acid, di(2-propylpentyl) ester (2.417\%), 7,9-Di-tert-butyl-1-oxaspiro $(4,5)$ deca-6,9-diene-2,8-dione (1.804\%), Benzoic acid, 3,5-dicyclohexyl-4hydroxy-, methyl ester (1.787\%) comprising major area.

Table 1: Antioxidant Activity of G. sylvestre from different locations.

\begin{tabular}{ccc}
\hline S.No & Sampling locations & Antioxidant Activity (\%) \\
\hline 1 & Shenbagadevi falls (Courtallam) & 73.40 \\
2 & Anthyodhaya sangham (Trichy) & 66.10 \\
3 & Vellingiri Hills (Coimbatore) & 65.90 \\
4 & Muniyankudisai Village (Arni) & 60.60 \\
5 & Kotta malai (Padavedu) & 54.80 \\
6 & Irulars Tribal Women's Welfare Society (Chengalpattu) & 49.30 \\
7 & Gandhi gram Trust (Dindigul) & 36.70 \\
\hline
\end{tabular}

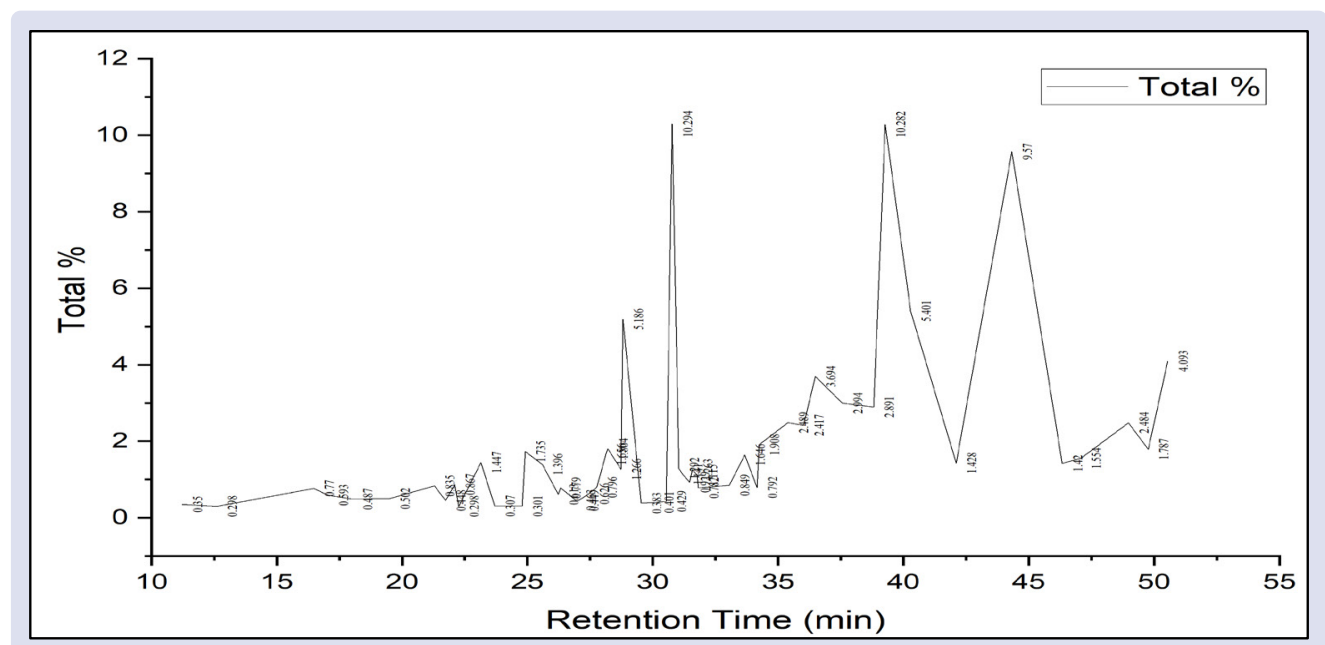

Figure 3: Abundance of the chemical constituents present in hexane extract from the G.sylvestre leaves. 
Table 2: Chemical composition of Hexane extract of Gymnema sylvestre from GCMS analysis.

\begin{tabular}{|c|c|c|c|c|c|}
\hline S. No & RT min & Name of compounds & Molecular formula & Molecular weight & $\%$ of total \\
\hline 1 & 11.219 & Undecane & $\mathrm{C} 11 \mathrm{H} 24$ & 156 & 0.350 \\
\hline 2 & 16.480 & Benzene, 1,3-bis(1,1-dimethylethyl)- & $\mathrm{C} 14 \mathrm{H} 22$ & 190 & 0.770 \\
\hline 3 & 17.040 & Dodecane, 2,6,11-trimethyl- & $\mathrm{C} 15 \mathrm{H} 32$ & 212 & 0.593 \\
\hline 4 & 18.014 & Pentadecane & $\mathrm{C} 15 \mathrm{H} 32$ & 212 & 0.487 \\
\hline 5 & 19.499 & Tetradecane & $\mathrm{C} 14 \mathrm{H} 30$ & 198 & 0.502 \\
\hline 6 & 21.740 & Phenol, 2,4-bis(1,1-dimethylethyl)- & $\mathrm{C} 14 \mathrm{H} 22 \mathrm{O}$ & 206 & 0.448 \\
\hline 7 & 22.263 & Tetradecane, 2,6,10-trimethyl- & $\mathrm{C} 17 \mathrm{H} 36$ & 240 & 0.298 \\
\hline 8 & 23.140 & Hexadecane & $\mathrm{C} 16 \mathrm{H} 34$ & 226 & 1.447 \\
\hline 9 & 23.700 & Octadecane, 1-chloro- & $\mathrm{C} 18 \mathrm{H} 37 \mathrm{Cl}$ & 288 & 0.307 \\
\hline 10 & 24.784 & Heptadecane & $\mathrm{C} 17 \mathrm{H} 36$ & 240 & 0.301 \\
\hline 11 & 24.918 & Tridecane, 2-methyl- & $\mathrm{C} 14 \mathrm{H} 30$ & 198 & 1.735 \\
\hline 12 & 25.600 & Hexadecane, 2,6,11,15-tetramethyl- & $\mathrm{C} 20 \mathrm{H} 42$ & 282 & 1.396 \\
\hline 13 & 26.233 & 1-Decanol, 2-hexyl- & $\mathrm{C} 16 \mathrm{H} 34 \mathrm{O}$ & 242 & 0.613 \\
\hline 14 & 26.915 & $3,7,11,15$-Tetramethyl-2-hexadecen-1-ol & $\mathrm{C} 20 \mathrm{H} 40 \mathrm{O}$ & 296 & 0.463 \\
\hline 15 & 27.037 & Heptadecane, 2,6,10,15-tetramethyl- & $\mathrm{C} 21 \mathrm{H} 44$ & 296 & 0.449 \\
\hline 16 & 27.451 & Dibutyl phthalate & $\mathrm{C} 16 \mathrm{H} 22 \mathrm{O} 4$ & 278 & 0.626 \\
\hline 17 & 27.767 & Ethanone, 2,2-dimethoxy-1,2-diphenyl- & $\mathrm{C} 16 \mathrm{H} 16 \mathrm{O} 3$ & 256 & 0.796 \\
\hline 18 & 28.133 & Nonadecane, 2-methyl- & $\mathrm{C} 20 \mathrm{H} 42$ & 282 & 1.656 \\
\hline 19 & 28.206 & $\begin{array}{l}\text { 7,9-Di-tert-butyl-1-oxaspiro }(4,5) \text { deca-6,9-diene-2,8- } \\
\text { dione }\end{array}$ & $\mathrm{C} 17 \mathrm{H} 24 \mathrm{O} 3$ & 276 & 1.804 \\
\hline 20 & 28.814 & n-Hexadecanoic acid & $\mathrm{C} 16 \mathrm{H} 32 \mathrm{O} 2$ & 256 & 5.186 \\
\hline 21 & 29.533 & Ethanol, 2-(9-octadecenyloxy)-, (Z)- & $\mathrm{C} 20 \mathrm{H} 40 \mathrm{O} 2$ & 312 & 0.383 \\
\hline 22 & 30.531 & Heneicosane & $\mathrm{C} 21 \mathrm{H} 44$ & 296 & 0.429 \\
\hline 23 & 30.763 & Phytol & $\mathrm{C} 20 \mathrm{H} 40 \mathrm{O}$ & 296 & 10.294 \\
\hline 24 & 31.043 & Heptadecane, 2-methyl- & $\mathrm{C} 18 \mathrm{H} 38$ & 254 & 1.292 \\
\hline 25 & 31.189 & Z-(13,14-Epoxy)tetradec-11-en-1-ol acetate & $\mathrm{C} 16 \mathrm{H} 28 \mathrm{O} 3$ & 268 & 1.141 \\
\hline 26 & 31.469 & Octadecane, 3-ethyl-5-(2-ethylbutyl)- & $\mathrm{C} 26 \mathrm{H} 54$ & 366 & 0.929 \\
\hline 27 & 31.578 & Eicosane, 2-methyl- & $\mathrm{C} 21 \mathrm{H} 44$ & 296 & 1.263 \\
\hline 28 & 31.761 & $\mathrm{n}$-Tetracosanol-1 & $\mathrm{C} 24 \mathrm{H} 50 \mathrm{O}$ & 354 & 1.115 \\
\hline 29 & 33.673 & 1-Cyclohexyldimethylsilyloxy-3,5-dimethylbenzene & $\mathrm{C} 16 \mathrm{H} 26 \mathrm{OSi}$ & 262 & 1.646 \\
\hline 30 & 36.011 & Phthalic acid, di(2-propylpentyl) ester & $\mathrm{C} 24 \mathrm{H} 38 \mathrm{O} 4$ & 390 & 2.417 \\
\hline 31 & 39.274 & Squalene & $\mathrm{C} 30 \mathrm{H} 50$ & 410 & 10.282 \\
\hline 32 & 44.315 & Tetratriacontane & $\mathrm{C} 34 \mathrm{H} 70$ & 478 & 9.570 \\
\hline 33 & 46.336 & Lup-20(29)-en-3-one & $\mathrm{C} 30 \mathrm{H} 48 \mathrm{O}$ & 424 & 1.420 \\
\hline 34 & 47.079 & Tetracosane, 11-decyl- & $\mathrm{C} 34 \mathrm{H} 70$ & 478 & 1.554 \\
\hline 35 & 48.979 & Stigmasterol & $\mathrm{C} 29 \mathrm{H} 48 \mathrm{O}$ & 412 & 2.484 \\
\hline 36 & 49.758 & Benzoic acid, 3,5-dicyclohexyl-4-hydroxy-, methyl ester & $\mathrm{C} 20 \mathrm{H} 28 \mathrm{O} 3$ & 316 & 1.787 \\
\hline
\end{tabular}

34 compounds were identified by GC-MS analysis (Figure 4 ) in the benzene extract of G.sylvestre. The compounds which occupied the major percentage in the extract are Eicosane $(>20 \%)$, Tetratriacontane (>19 \%), Hexadecane, 2,6,11,15-tetramethyl- (>5\%), Benzoic acid, 3,5-dicyclohexyl-4-hydroxy-, methyl ester (5.515\%),Tetracosane (4.803\%), Squalene (4.797\%), Ethylbenzene (3.052\%), Hexadecane (2.862 \%), Phytol (2.788 \%), n-Hexadecanoic acid (2.622 \%) (Table 3).

The GC-MS analysis (Figure 5) results of the chloroform extract of G.sylvestre showed the presence of 32 compounds in it. The compounds present in the chloroform extract is given in the Table 4. Among the compounds identified Eicosane (>14\%), Phytol (8.667\%), Heptadecane, 9-hexyl- (>7 \%), Squalene (5.441\%), 7,9-Di-tert-butyl-1-oxaspiro(4,5) deca-6,9-diene-2,8-dione (5.074\%), n-Hexadecanoic acid (5.005\%), Tetracosane (4.469\%), Hexadecane (>4 \%), Stigmasterol (3.879 \%), Tetratriacontane $(3.717 \%)$, Phthalic acid, hex-3-yl isobutyl ester (3.439 $\%)$, Octadecane, 3-ethyl-5-(2-ethylbutyl)- $(2.720 \%)$ represented more than $67 \%$ of the total compounds.

The ethyl acetate G.sylvestre crude extract on GCMS analysis (Figure 6) revealed the presence of 43 different organic compounds listed in the Table 5 with major compounds as follows: Eicosane, 2-methyl( $>9 \%)$, n-Tetracosanol-1 (6.579\%), Heneicosane ( $>6 \%)$, Fumaric acid, 2-chloroethyl hexadecyl ester (6.100\%), Tetracosane ( $>5$ $\%)$, Eicosane (>3 \%), E-15-Heptadecenal (3.801 \%), Hexadecane, 2,6,11,15-tetramethyl- (3.582\%), Triacontane, 1-bromo (3.258\%), Ethanone, 2,2-dimethoxy-1,2-diphenyl- (2.964\%), Phenol, 2,4-bis(1,1dimethylethyl)- (2.770 \%), Tetracosane (2.266 \%), Cetene (1.883\%).

The GC-MS analysis (Figure 7) of the ethanolic G.sylvestre leaves extract based on the retention time on capillary column fused with silica is listed in the Table 6 with major compounds as 2-Pentanone, 3,3,4,4-tetramethyl (15.885\%), Squalene (15.075\%), n-Hexadecanoic acid (7.086\%), Phytol (6.351\%), Cholesterol (5.966\%), Octadecane, 1,1'-[(1-methyl-1,2-ethanediyl) bis(oxy)] bis- (5.218\%), Stigmasterol (4.314\%), (E)-9-Octadecenoic acid ethyl ester (4.290\%), trans-13Octadecenoic acid (4.158\%), 1,2,3,4-Cyclohexanetetrol (3.699\%), Hexadecanoic acid, ethyl ester (3.084\%), Eicosanoic acid (2.859\%), Tetraethyl silicate $(2.804 \%)$. From the MS chromatogram, a total of 29 compounds were identified. These compounds are members of different types of organic groups such as alcohols, amines, fatty acids, terpenes.

The methanol plant extract was analysed using GC-MS (Figure 8). A total of 17 different compounds which is listed in the Table 7 where compounds comprising major percentage are 2-Pentanone, 3,3,4,4-tetramethyl- (61.21\%), Inositol, 1-deoxy- (21.218\%), 
Table 3: Chemical composition of Benzene extract of Gymnema sylvestre from GCMS analysis.

\begin{tabular}{|c|c|c|c|c|c|}
\hline S. No & RT min & Name of compounds & Molecular formula & Molecular weight & $\%$ of total \\
\hline 1 & 4.400 & Ethylbenzene & $\mathrm{C} 8 \mathrm{H} 10$ & 106 & 3.052 \\
\hline 2 & 4.583 & Benzene, 1,3-dimethyl- & $\mathrm{C} 8 \mathrm{H} 10$ & 106 & 1.406 \\
\hline 3 & 15.213 & Dodecane & $\mathrm{C} 12 \mathrm{H} 26$ & 170 & 0.478 \\
\hline 4 & 16.504 & Benzene, 1,3-bis(1,1-dimethylethyl)- & $\mathrm{C} 14 \mathrm{H} 22$ & 190 & 1.636 \\
\hline 5 & 16.686 & 2,4-Dimethyldodecane & $\mathrm{C} 14 \mathrm{H} 30$ & 198 & 0.186 \\
\hline 6 & 17.052 & Dodecane, 2,7,10-trimethyl- & $\mathrm{C} 15 \mathrm{H} 32$ & 212 & 0.648 \\
\hline 7 & 18.026 & Dodecane, 2,6,11-trimethyl- & $\mathrm{C} 15 \mathrm{H} 32$ & 212 & 0.225 \\
\hline 8 & 19.511 & Tetradecane & $\mathrm{C} 14 \mathrm{H} 30$ & 198 & 1.701 \\
\hline 9 & 21.301 & Heptadecane & $\mathrm{C} 17 \mathrm{H} 36$ & 240 & 1.329 \\
\hline 10 & 21.800 & Phenol, 2,4-bis(1,1-dimethylethyl)- & $\mathrm{C} 14 \mathrm{H} 22 \mathrm{O}$ & 206 & 0.492 \\
\hline 11 & 23.128 & Hexadecane & $\mathrm{C} 16 \mathrm{H} 34$ & 226 & 2.862 \\
\hline 12 & 26.221 & E-15-Heptadecenal & $\mathrm{C} 17 \mathrm{H} 32 \mathrm{O}$ & 252 & 0.879 \\
\hline 13 & 26.915 & 3,7,11,15-Tetramethyl-2-hexadecen-1-ol & $\mathrm{C} 20 \mathrm{H} 40 \mathrm{O}$ & 296 & 0.416 \\
\hline 14 & 27.463 & Dibutyl phthalate & $\mathrm{C} 16 \mathrm{H} 2204$ & 278 & 0.430 \\
\hline 15 & 27.767 & Ethanone, 2,2-dimethoxy-1,2-diphenyl- & $\mathrm{C} 16 \mathrm{H} 16 \mathrm{O} 3$ & 256 & 1.355 \\
\hline 16 & 27.974 & Octadecane, 3-ethyl-5-(2-ethylbutyl)- & $\mathrm{C} 26 \mathrm{H} 54$ & 366 & 0.429 \\
\hline 17 & 28.132 & Hexadecane, 2,6,11,15-tetramethyl- & $\mathrm{C} 20 \mathrm{H} 42$ & 282 & 2.466 \\
\hline 18 & 28.839 & n-Hexadecanoic acid & $\mathrm{C} 16 \mathrm{H} 32 \mathrm{O} 2$ & 256 & 2.622 \\
\hline 19 & 29.119 & 10-Heneicosene $(c, t)$ & $\mathrm{C} 21 \mathrm{H} 42$ & 294 & 0.502 \\
\hline 20 & 30.020 & Nonadecane, 2-methyl- & $\mathrm{C} 20 \mathrm{H} 42$ & 282 & 0.555 \\
\hline 21 & 30.543 & Heneicosane & $\mathrm{C} 21 \mathrm{H} 44$ & 296 & 0.956 \\
\hline 22 & 30.762 & Phytol & $\mathrm{C} 20 \mathrm{H} 40 \mathrm{O}$ & 296 & 2.788 \\
\hline 23 & 31.042 & Eicosane, 2-methyl- & $\mathrm{C} 21 \mathrm{H} 44$ & 296 & 1.902 \\
\hline 24 & 31.469 & Eicosane, 7-hexyl- & $\mathrm{C} 26 \mathrm{H} 54$ & 366 & 0.500 \\
\hline 25 & 31.761 & 1-Heneicosyl formate & $\mathrm{C} 22 \mathrm{H} 44 \mathrm{O}$ & 340 & 1.491 \\
\hline 26 & 33.673 & 1-Cyclohexyldimethylsilyloxy-3,5-dimethylbenzene & $\mathrm{C} 16 \mathrm{H} 26 \mathrm{OSi}$ & 262 & 2.367 \\
\hline 27 & 34.245 & Tetracosane & $\mathrm{C} 24 \mathrm{H} 50$ & 338 & 4.803 \\
\hline 28 & 36.023 & Heneicosane, 11-(1-ethylpropyl)- & $\mathrm{C} 26 \mathrm{H} 54$ & 366 & 0.793 \\
\hline 29 & 36.485 & Eicosane & $\mathrm{C} 20 \mathrm{H} 42$ & 282 & 7.272 \\
\hline 30 & 37.569 & Tetratriacontane & $\mathrm{C} 34 \mathrm{H} 70$ & 478 & 5.102 \\
\hline 31 & 39.274 & Squalene & $\mathrm{C} 30 \mathrm{H} 50$ & 410 & 4.797 \\
\hline 32 & 47.607 & Tetracosane, 11-decyl- & $\mathrm{C} 34 \mathrm{H} 70$ & 478 & 0.674 \\
\hline 33 & 48.966 & Stigmasterol & $\mathrm{C} 29 \mathrm{H} 48 \mathrm{O}$ & 412 & 1.101 \\
\hline 34 & 49.782 & Benzoic acid, 3,5-dicyclohexyl-4-hydroxy-, methyl ester & $\mathrm{C} 20 \mathrm{H} 28 \mathrm{O} 3$ & 316 & 5.515 \\
\hline
\end{tabular}

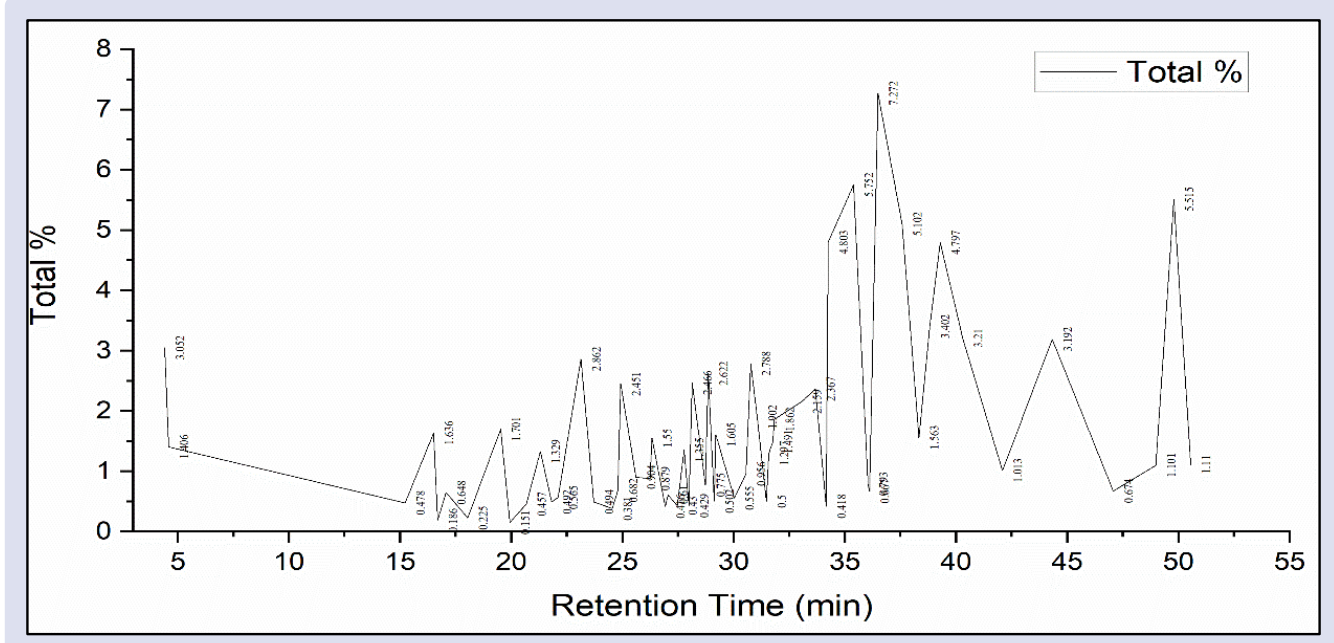

Figure 4: Abundance of the chemical constituents present in benzene extract from the G.sylvestre leaves. 
Table 4: Chemical composition of Chloroform extract of Gymnema sylvestre from GCMS analysis.

\begin{tabular}{|c|c|c|c|c|c|}
\hline S. No & RT Min & Name of the compounds & Molecular formula & Molecular weight & $\%$ of total \\
\hline 1 & 16.504 & Benzene, 1,3-bis(1,1-dimethylethyl)- & $\mathrm{C} 14 \mathrm{H} 22$ & 190 & 1.221 \\
\hline 2 & 19.511 & Tetradecane & $\mathrm{C} 14 \mathrm{H} 30$ & 198 & 0.728 \\
\hline 3 & 21.301 & Heptadecane, 2,6,10,15-tetramethyl- & $\mathrm{C} 21 \mathrm{H} 44$ & 296 & 0.934 \\
\hline 4 & 21.788 & Phenol, 2,4-bis(1,1-dimethylethyl)- & $\mathrm{C} 14 \mathrm{H} 22 \mathrm{O}$ & 206 & 0.955 \\
\hline 5 & 22.098 & Dodecane, 2,6,11-trimethyl- & $\mathrm{C} 15 \mathrm{H} 32$ & 212 & 1.095 \\
\hline 6 & 23.110 & Hexadecane & $\mathrm{C} 16 \mathrm{H} 34$ & 226 & 3.781 \\
\hline 7 & 23.700 & Benzeneacetic acid, 4-tetradecyl ester & $\mathrm{C} 22 \mathrm{H} 36 \mathrm{O} 2$ & 332 & 0.555 \\
\hline 8 & 23.931 & Benzene, (1-propyloctyl)- & $\mathrm{C} 17 \mathrm{H} 28$ & 232 & 0.808 \\
\hline 9 & 24.930 & Nonadecane, 2-methyl- & $\mathrm{C} 20 \mathrm{H} 42$ & 282 & 2.383 \\
\hline 10 & 25.271 & Benzene, (1-pentylheptyl)- & $\mathrm{C} 18 \mathrm{H} 30$ & 246 & 0.534 \\
\hline 11 & 25.612 & Heptadecane, 2,6,10,15-tetramethyl- & $\mathrm{C} 21 \mathrm{H} 44$ & 296 & 2.091 \\
\hline 12 & 27.049 & 1-Chloroeicosane & $\mathrm{C} 20 \mathrm{H} 41 \mathrm{Cl}$ & 316 & 0.866 \\
\hline 13 & 27.450 & Phthalic acid, hex-3-yl isobutyl ester & $\mathrm{C} 18 \mathrm{H} 26 \mathrm{O} 4$ & 306 & 3.439 \\
\hline 14 & 27.767 & Ethanone, 2,2-dimethoxy-1,2-diphenyl- & $\mathrm{C} 16 \mathrm{H} 16 \mathrm{O} 3$ & 256 & 1.942 \\
\hline 15 & 28.145 & 2-methyloctacosane & $\mathrm{C} 29 \mathrm{H} 60$ & 408 & 1.502 \\
\hline 16 & 28.205 & $\begin{array}{l}\text { 7,9-Di-tert-butyl-1-oxaspiro }(4,5) \text { deca-6,9-diene-2,8- } \\
\text { dione }\end{array}$ & $\mathrm{C} 17 \mathrm{H} 24 \mathrm{O} 3$ & 276 & 5.074 \\
\hline 17 & 28.826 & n-Hexadecanoic acid & $\mathrm{C} 16 \mathrm{H} 32 \mathrm{O} 2$ & 256 & 5.005 \\
\hline 18 & 29.204 & Hexadecanoic acid, ethyl ester & $\mathrm{C} 18 \mathrm{H} 36 \mathrm{O} 2$ & 284 & 2.189 \\
\hline 19 & 30.775 & Phytol & $\mathrm{C} 20 \mathrm{H} 40 \mathrm{O}$ & 296 & 8.667 \\
\hline 20 & 31.043 & Eicosane, 2-methyl- & $\mathrm{C} 21 \mathrm{H} 44$ & 296 & 1.283 \\
\hline 21 & 31.481 & Octadecanoic acid & $\mathrm{C} 18 \mathrm{H} 36 \mathrm{O} 2$ & 284 & 0.751 \\
\hline 22 & 31.773 & 1-Decanol, 2-hexyl- & $\mathrm{C} 16 \mathrm{H} 32 \mathrm{O}$ & 242 & 1.016 \\
\hline 23 & 32.808 & 1-Cyclohexyldimethylsilyloxy-3,5-dimethylbenzene & $\mathrm{C} 16 \mathrm{H} 26 \mathrm{OSi}$ & 262 & 0.982 \\
\hline 24 & 33.673 & 1-Cyclohexyldimethylsilyloxy-3,5-dimethylbenzene & $\mathrm{C} 16 \mathrm{H} 26 \mathrm{OSi}$ & 262 & 2.526 \\
\hline 25 & 34.257 & Tetracosane & $\mathrm{C} 24 \mathrm{H} 50$ & 338 & 4.469 \\
\hline 26 & 37.581 & Octadecane, 3-ethyl-5-(2-ethylbutyl)- & $\mathrm{C} 26 \mathrm{H} 54$ & 366 & 2.720 \\
\hline 27 & 39.298 & Squalene & $\mathrm{C} 30 \mathrm{H} 50$ & 410 & 5.441 \\
\hline 28 & 40.309 & Heptadecane, 9-hexyl- & $\mathrm{C} 23 \mathrm{H} 48$ & 324 & 3.933 \\
\hline 29 & 42.009 & Octadecanoic acid, 2-(hexadecyloxy)ethyl ester & $\mathrm{C} 36 \mathrm{H} 72 \mathrm{O} 3$ & 552 & 1.736 \\
\hline 30 & 44.339 & Eicosane & $\mathrm{C} 2 \mathrm{OH} 42$ & 282 & 7.575 \\
\hline 31 & 49.015 & Stigmasterol & $\mathrm{C} 29 \mathrm{H} 48 \mathrm{O}$ & 412 & 3.879 \\
\hline 32 & 50.561 & Tetratriacontane & $\mathrm{C} 34 \mathrm{H} 70$ & 478 & 3.717 \\
\hline
\end{tabular}

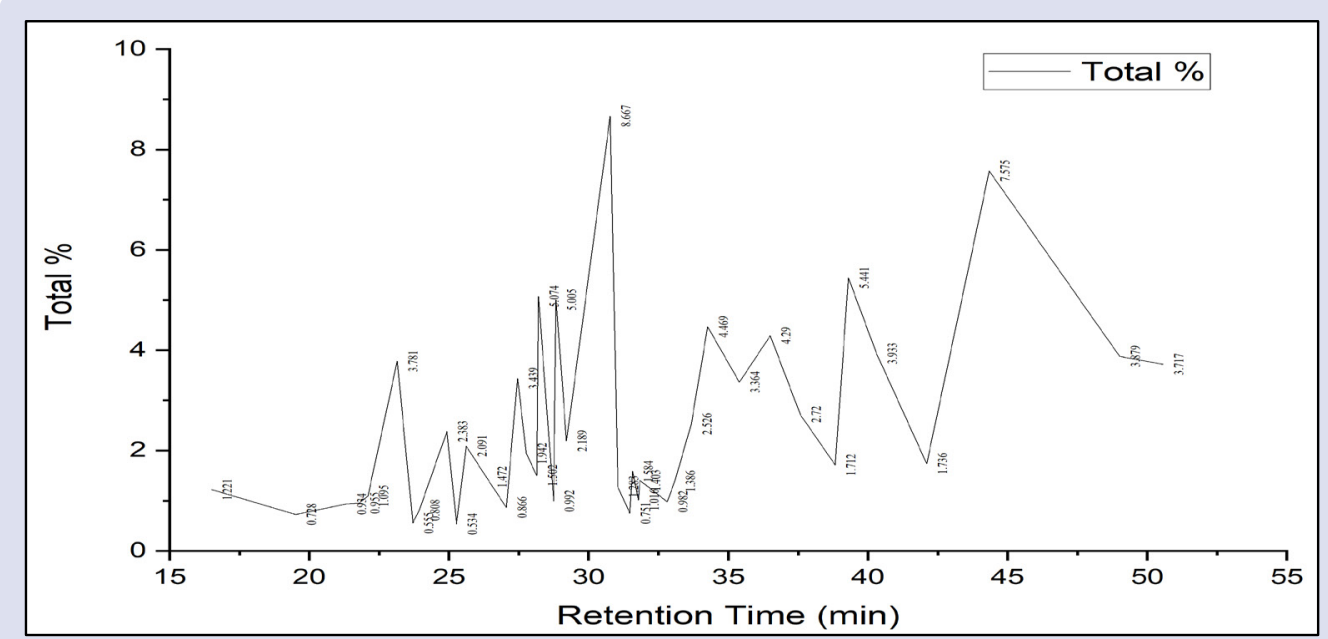

Figure 5: Abundance of the chemical constituents present in chloroform extract from the G.sylvestre leaves. 
Table 5: Chemical composition of Ethyl acetate extract of Gymnema sylvestre from GCMS analysis.

\begin{tabular}{|c|c|c|c|c|c|}
\hline S. No & RT min & Name of compounds & Molecular formula & Molecular weight & $\%$ of total \\
\hline 1 & 32.808 & 1-Cyclohexyldimethylsilyloxy-3,5-dimethylbenzene & $\mathrm{C} 16 \mathrm{H} 26 \mathrm{OSi}$ & 262 & 0.707 \\
\hline 2 & 26.915 & 3,7,11,15-Tetramethyl-2-hexadecen-1-ol & $\mathrm{C} 20 \mathrm{H} 40 \mathrm{O}$ & 296 & 0.419 \\
\hline 3 & 13.094 & 4-Piperidinone, 2,2,6,6-tetramethyl- & $\mathrm{C} 9 \mathrm{H} 17 \mathrm{NO}$ & 155 & 0.447 \\
\hline 4 & 29.119 & 5-Eicosene, (E)- & $\mathrm{C} 20 \mathrm{H} 40$ & 280 & 1.708 \\
\hline 5 & 16.492 & Benzene, 1,3-bis(1,1-dimethylethyl)- & $\mathrm{C} 14 \mathrm{H} 22$ & 190 & 0.617 \\
\hline 6 & 49.770 & Benzoic acid, 3,5-dicyclohexyl-4-hydroxy-, methyl ester & $\mathrm{C} 20 \mathrm{H} 28 \mathrm{O} 3$ & 316 & 2.792 \\
\hline 7 & 23.128 & Cetene & $\mathrm{C} 16 \mathrm{H} 32$ & 224 & 1.883 \\
\hline 8 & 23.018 & Dodecane, 2,6,11-trimethyl- & $\mathrm{C} 15 \mathrm{H} 32$ & 212 & 0.729 \\
\hline 9 & 26.221 & E-15-Heptadecenal & $\mathrm{C} 17 \mathrm{H} 32 \mathrm{O}$ & 252 & 3.801 \\
\hline 10 & 35.390 & Eicosane & $\mathrm{C} 20 \mathrm{H} 42$ & 282 & 1.909 \\
\hline 11 & 28.132 & Eicosane, 2-methyl- & $\mathrm{C} 21 \mathrm{H} 44$ & 296 & 4.922 \\
\hline 12 & 34.172 & Eicosane, 7-hexyl- & $\mathrm{C} 26 \mathrm{H} 54$ & 366 & 1.174 \\
\hline 13 & 27.767 & Ethanone, 2,2-dimethoxy-1,2-diphenyl- & $\mathrm{C} 16 \mathrm{H} 16 \mathrm{O} 3$ & 256 & 2.964 \\
\hline 14 & 33.673 & Fumaric acid, 2-chloroethyl hexadecyl ester & $\mathrm{C} 22 \mathrm{H} 39 \mathrm{ClO} 4$ & 402 & 6.100 \\
\hline 15 & 30.531 & Heneicosane & $\mathrm{C} 21 \mathrm{H} 44$ & 296 & 1.908 \\
\hline 16 & 41.697 & Heneicosane, 11-(1-ethylpropyl)- & $\mathrm{C} 26 \mathrm{H} 54$ & 306 & 0.743 \\
\hline 17 & 24.772 & Heptadecane & $\mathrm{C} 17 \mathrm{H} 36$ & 240 & 0.529 \\
\hline 18 & 21.289 & Heptadecane, 2,6,10,15-tetramethyl- & $\mathrm{C} 21 \mathrm{H} 44$ & 296 & 0.998 \\
\hline 19 & 46.738 & Heptadecane, 9-hexyl- & $\mathrm{C} 23 \mathrm{H} 48$ & 324 & 0.733 \\
\hline 20 & 30.665 & Heptadecane, 9-octyl- & $\mathrm{C} 25 \mathrm{H} 52$ & 353 & 0.658 \\
\hline 21 & 25.052 & Hexadecane & $\mathrm{C} 16 \mathrm{H} 34$ & 226 & 0.465 \\
\hline 22 & 24.918 & Hexadecane, 2,6,11,15-tetramethyl- & $\mathrm{C} 20 \mathrm{H} 42$ & 282 & 3.582 \\
\hline 23 & 34.635 & i-Propyl 5,9,19-octacosatrienoate & $\mathrm{C} 31 \mathrm{H} 56 \mathrm{O} 2$ & 460 & 0.749 \\
\hline 24 & 28.839 & n-Hexadecanoic acid & $\mathrm{C} 16 \mathrm{H} 32 \mathrm{O} 2$ & 256 & 0.662 \\
\hline 25 & 30.020 & Nonadecane, 2-methyl- & $\mathrm{C} 20 \mathrm{H} 42$ & 282 & 1.369 \\
\hline 26 & 31.761 & $\mathrm{n}$-Tetracosanol-1 & $\mathrm{C} 24 \mathrm{H} 50 \mathrm{O}$ & 354 & 6.579 \\
\hline 27 & 29.545 & Octadecanal & $\mathrm{C} 18 \mathrm{H} 36 \mathrm{O}$ & 268 & 0.857 \\
\hline 28 & 26.306 & Octadecane & $\mathrm{C} 18 \mathrm{H} 38$ & 252 & 1.611 \\
\hline 29 & 27.962 & Octadecane, 2-methyl- & $\mathrm{C} 19 \mathrm{H} 40$ & 268 & 0.606 \\
\hline 30 & 50.525 & Octadecane, 3-ethyl-5-(2-ethylbutyl)- & $\mathrm{C} 26 \mathrm{H} 54$ & 366 & 0.791 \\
\hline 31 & 21.740 & Phenol, 2,4-bis(1,1-dimethylethyl)- & $\mathrm{C} 14 \mathrm{H} 22 \mathrm{O}$ & 206 & 2.770 \\
\hline 32 & 30.775 & Phytol & $\mathrm{C} 20 \mathrm{H} 40 \mathrm{O}$ & 296 & 1.883 \\
\hline 33 & 39.274 & Squalene & $\mathrm{C} 30 \mathrm{H} 50$ & 410 & 1.698 \\
\hline 34 & 48.966 & Stigmasterol & $\mathrm{C} 29 \mathrm{H} 48 \mathrm{O}$ & 412 & 0.784 \\
\hline 35 & 36.486 & Tetracosane & $\mathrm{C} 24 \mathrm{H} 50$ & 338 & 2.266 \\
\hline 36 & 36.023 & Tetracosane, 11-decyl- & C34H70 & 478 & 2.550 \\
\hline 37 & 19.511 & Tetradecane & $\mathrm{C} 14 \mathrm{H} 30$ & 198 & 0.742 \\
\hline 38 & 27.536 & Tetradecane, 2,6,10-trimethyl- & $\mathrm{C} 17 \mathrm{H} 36$ & 240 & 0.788 \\
\hline 39 & 28.729 & Tetradecane, 2-methyl- & $\mathrm{C} 15 \mathrm{H} 32$ & 212 & 1.499 \\
\hline 40 & 40.285 & Tetratriacontane & $\mathrm{C} 34 \mathrm{H} 70$ & 478 & 0.236 \\
\hline 41 & 36.108 & Triacontane, 1-bromo- & $\mathrm{C} 30 \mathrm{H} 61 \mathrm{Br}$ & 500 & 3.258 \\
\hline 42 & 33.064 & Tricosane & $\mathrm{C} 23 \mathrm{H} 48$ & 324 & 0.782 \\
\hline 43 & 24.540 & Tridecane, 2-methyl- & $\mathrm{C} 14 \mathrm{H} 30$ & 198 & 0.596 \\
\hline
\end{tabular}


Table 6: Chemical composition of Ethanol extract of Gymnema sylvestre from GCMS analysis.

\begin{tabular}{|c|c|c|c|c|c|}
\hline S. No & RT min & Name of the compound & Molecular formula & Molecular weight & $\%$ of total \\
\hline 1 & 7.907 & Disiloxane, 1,3-diethoxy-1,1,3,3-tetramethyl- & $\mathrm{C} 8 \mathrm{H} 22 \mathrm{O} 3 \mathrm{Si} 2$ & 222 & 0.174 \\
\hline 2 & 9.113 & Tetraethyl silicate & $\mathrm{C} 8 \mathrm{H} 20 \mathrm{O} 4 \mathrm{Si}$ & 208 & 2.804 \\
\hline 3 & 12.571 & d-Mannitol, 1-decylsulfonyl- & $\mathrm{C} 16 \mathrm{H} 34 \mathrm{O} 7 \mathrm{~S}$ & 370 & 0.173 \\
\hline 4 & 15.006 & 3-Dodecene, (E)- & $\mathrm{C} 12 \mathrm{H} 24$ & 168 & 0.554 \\
\hline 5 & 16.492 & Benzene, 1,3-bis(1,1-dimethylethyl)- & $\mathrm{C} 14 \mathrm{H} 22$ & 190 & 0.256 \\
\hline 6 & 19.353 & 4-Trifluoroacetoxytetradecane & $\mathrm{C} 16 \mathrm{H} 29 \mathrm{~F} 3 \mathrm{O} 2$ & 310 & 0.389 \\
\hline 7 & 19.499 & 2-Hexyl-1-octanol & $\mathrm{C} 14 \mathrm{H} 30 \mathrm{O}$ & 214 & 0.830 \\
\hline 8 & 21.289 & Tetradecane, 2,6,10-trimethyl- & $\mathrm{C} 17 \mathrm{H} 36$ & 240 & 0.219 \\
\hline 9 & 21.764 & Phenol, 2,4-bis(1,1-dimethylethyl)- & $\mathrm{C} 14 \mathrm{H} 22 \mathrm{O}$ & 206 & 0.909 \\
\hline 10 & 22.312 & 1,2,3,4-Cyclohexanetetrol & $\mathrm{C} 6 \mathrm{H} 12 \mathrm{O} 4$ & 148 & 3.699 \\
\hline 11 & 22.811 & 2-Pentanone, 3,3,4,4-tetramethyl- & $\mathrm{C} 9 \mathrm{H} 18 \mathrm{O}$ & 142 & 15.885 \\
\hline 12 & 26.221 & 2-Dodecanol & $\mathrm{C} 12 \mathrm{H} 26 \mathrm{O}$ & 186 & 0.531 \\
\hline 13 & 26.902 & 3,7,11,15-Tetramethyl-2-hexadecen-1-ol & $\mathrm{C} 20 \mathrm{H} 40 \mathrm{O}$ & 296 & 1.139 \\
\hline 14 & 28.802 & n-Hexadecanoic acid & $\mathrm{C} 16 \mathrm{H} 32 \mathrm{O} 2$ & 256 & 7.086 \\
\hline 15 & 29.143 & Hexadecanoic acid, ethyl ester & $\mathrm{C} 18 \mathrm{H} 36 \mathrm{O} 2$ & 284 & 3.084 \\
\hline 16 & 30.762 & Phytol & $\mathrm{C} 20 \mathrm{H} 40 \mathrm{O}$ & 296 & 6.351 \\
\hline 17 & 31.298 & trans-13-Octadecenoic acid & $\mathrm{C} 18 \mathrm{H} 34 \mathrm{O} 2$ & 282 & 4.158 \\
\hline 18 & 31.456 & (E)-9-Octadecenoic acid ethyl ester & $\mathrm{C} 20 \mathrm{H} 38 \mathrm{O} 2$ & 310 & 4.290 \\
\hline 19 & 31.749 & Eicosanoic acid & $\mathrm{C} 20 \mathrm{H} 40 \mathrm{O} 2$ & 312 & 2.859 \\
\hline 20 & 33.052 & Octadecane, 3-ethyl-5-(2-ethylbutyl)- & $\mathrm{C} 26 \mathrm{H} 54$ & 366 & 0.564 \\
\hline 21 & 33.746 & $\begin{array}{c}\text { 9,12,15-Octadecatrienoic acid, } 2,3-\text { bis }[\text { (trimethylsilyl) } \\
\text { oxy propyl ester, }(\mathrm{Z}, \mathrm{Z}, \mathrm{Z}) \text { - }\end{array}$ & $\mathrm{C} 27 \mathrm{H} 52 \mathrm{O} 4 \mathrm{Si} 2$ & 496 & 0.802 \\
\hline 22 & 34.245 & Heptadecane, 9-hexyl- & $\mathrm{C} 23 \mathrm{H} 48$ & 324 & 1.503 \\
\hline 23 & 34.975 & N1-Benzyl-N2(bezylidenyl-benzylamino)-benzamidin & $\mathrm{C} 28 \mathrm{H} 25 \mathrm{~N} 3$ & 403 & 0.971 \\
\hline 24 & 35.389 & Docosane, 11-butyl- & $\mathrm{C} 26 \mathrm{H} 54$ & 366 & 1.003 \\
\hline 25 & 36.485 & Tetracosane, 11-decyl- & $\mathrm{C} 34 \mathrm{H} 70$ & 478 & 1.178 \\
\hline 26 & 36.814 & Cholesterol & $\mathrm{C} 27 \mathrm{H} 46 \mathrm{O}$ & 386 & 5.966 \\
\hline 27 & 39.274 & Squalene & $\mathrm{C} 30 \mathrm{H} 50$ & 410 & 15.075 \\
\hline 28 & 44.303 & $\begin{array}{c}\text { Octadecane, } 1,1 \text { '-[(1-methyl-1,2-ethanediyl) bis(oxy) }] \\
\text { bis- }\end{array}$ & C39H80O2 & 580 & 5.218 \\
\hline 29 & 48.954 & Stigmasterol & $\mathrm{C} 29 \mathrm{H} 48 \mathrm{O}$ & 412 & 4.314 \\
\hline
\end{tabular}

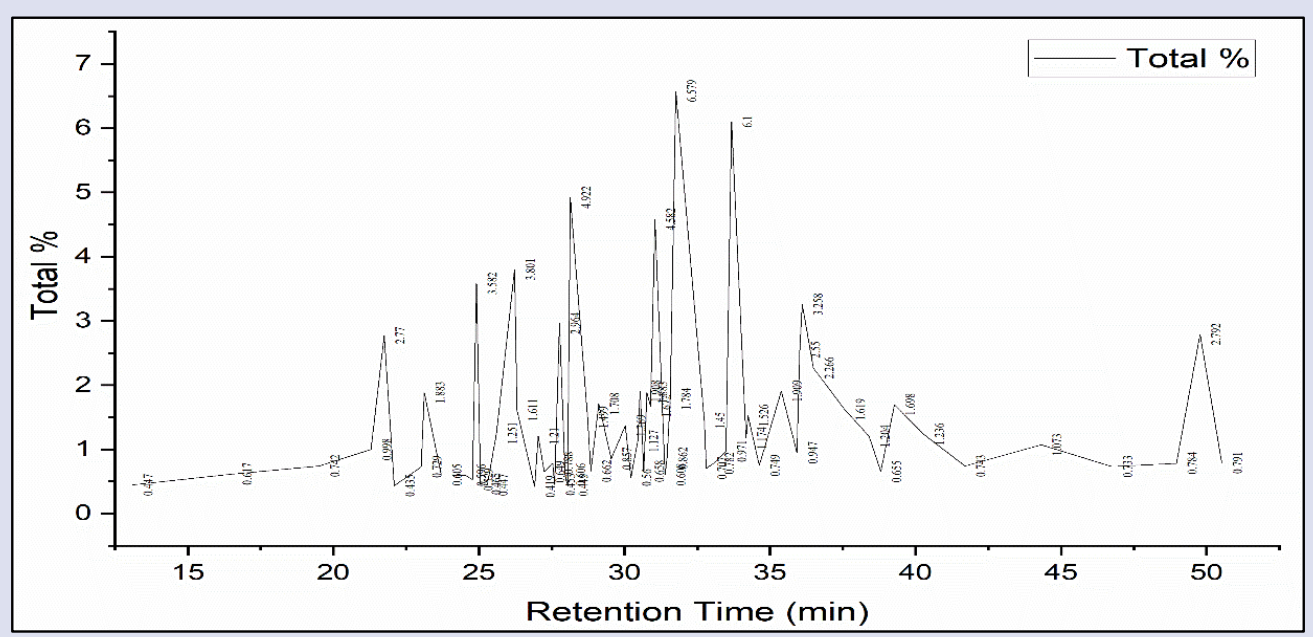

Figure 6: Abundance of the chemical constituents present in ethyl acetate extract from the G.sylvestre leaves. 


\section{Table 7: Chemical composition of Methanol extract of Gymnema sylvestre from GCMS analysis.}

\begin{tabular}{|c|c|c|c|c|c|}
\hline S. No & RT min & Name of compounds & Molecular formula & Molecular weight & $\%$ of total \\
\hline 1 & 9.088 & Decane & $\mathrm{C} 10 \mathrm{H} 22$ & 142 & 0.294 \\
\hline 2 & 12.547 & Undecane & $\mathrm{C} 11 \mathrm{H} 24$ & 156 & 0.237 \\
\hline 3 & 21.533 & 1,2,3,4-Cyclohexanetetrol & $\mathrm{C} 6 \mathrm{H} 12 \mathrm{O} 4$ & 148 & 3.768 \\
\hline 4 & 23.883 & 2-Pentanone, 3,3,4,4-tetramethyl- & $\mathrm{C} 9 \mathrm{H} 18 \mathrm{O}$ & 142 & 61.218 \\
\hline 5 & 26.209 & Inositol, 1-deoxy- & C6H12O5 & 164 & 21.218 \\
\hline 6 & 26.903 & $\begin{array}{l}\text { 3,7,11,15-Tetramethyl-2-hexadecen- } \\
\text { 1-ol }\end{array}$ & $\mathrm{C} 20 \mathrm{H} 40 \mathrm{O}$ & 296 & 0.912 \\
\hline 7 & 28.181 & Hexadecanoic acid, methyl ester & $\mathrm{C} 17 \mathrm{H} 34 \mathrm{O} 2$ & 270 & 0.195 \\
\hline 8 & 28.766 & n-Hexadecanoic acid & $\mathrm{C} 16 \mathrm{H} 32 \mathrm{O} 2$ & 256 & 3.616 \\
\hline 9 & 30.592 & 10-Octadecenoic acid, methyl ester & $\mathrm{C} 19 \mathrm{H} 36 \mathrm{O} 2$ & 296 & 0.187 \\
\hline 10 & 30.763 & Phytol & $\mathrm{C} 20 \mathrm{H} 40 \mathrm{O}$ & 296 & 1.693 \\
\hline 11 & 31.201 & 9,12,15-Octadecatrienoic acid, (Z,Z,Z)- & $\mathrm{C} 18 \mathrm{H} 30 \mathrm{O} 2$ & 278 & 2.521 \\
\hline 12 & 31.444 & Octadecanoic acid & $\mathrm{C} 18 \mathrm{H} 36 \mathrm{O} 2$ & 284 & 0.148 \\
\hline 13 & 33.356 & 2-Pyrrolidinone, 1-(9-octadecenyl)- & $\mathrm{C} 22 \mathrm{H} 41 \mathrm{NO}$ & 335 & 0.214 \\
\hline 14 & 35.645 & $\begin{array}{l}\text { Hexadecanoic acid, } \\
\text { 1-(hydroxymethyl)-1,2-ethanediyl } \\
\text { ester }\end{array}$ & $\mathrm{C} 35 \mathrm{H} 68 \mathrm{O} 5$ & 568 & 0.140 \\
\hline 15 & 36.011 & Phthalic acid, di(2-propylpentyl) ester & $\mathrm{C} 24 \mathrm{H} 38 \mathrm{O} 4$ & 390 & 0.128 \\
\hline 16 & 39.274 & Squalene & $\mathrm{C} 30 \mathrm{H} 50$ & 410 & 1.782 \\
\hline 17 & 48.954 & Stigmasterol & $\mathrm{C} 29 \mathrm{H} 48 \mathrm{O}$ & 412 & 0.860 \\
\hline
\end{tabular}

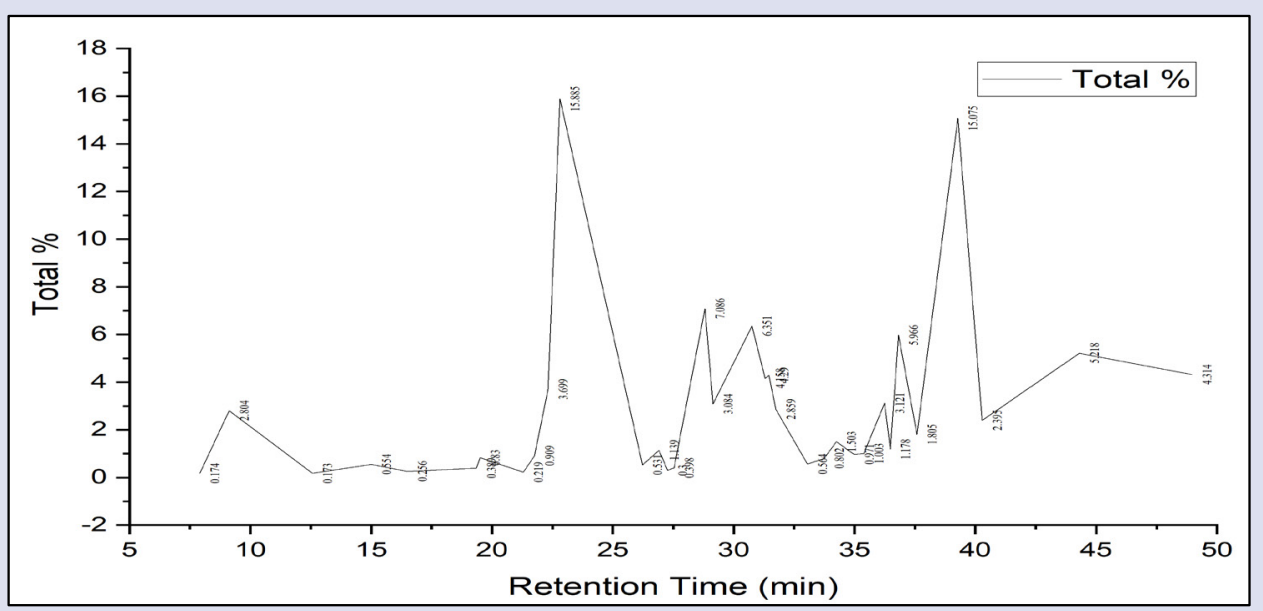

Figure 7: Abundance of the chemical constituents present in ethanol extract from the G.sylvestre leaves.

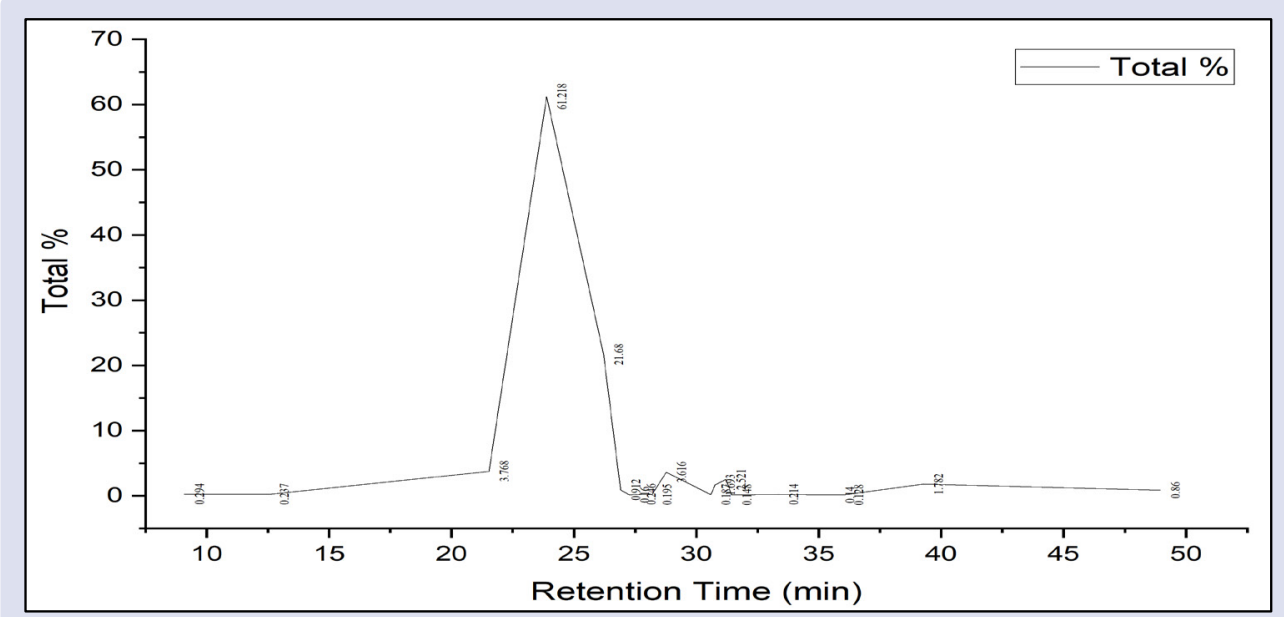

Figure 8: Abundance of the chemical constituents present in methanol extract from the G.sylvestre leaves. 
1,2,3,4-Cyclohexanetetrol (3.768\%), n-Hexadecanoic acid (3.616), 9,12,15-Octadecatrienoic acid, (Z,Z,Z)- (2.521\%), Squalene (1.782\%), Phytol (1.693 \%)

\section{DISCUSSION}

In the current study, out of various locations (Districts of Tamilnadu), the plant samples collected from Shenbagadevi falls, Courtallam showed maximum antioxidant activity and it was found to be in the order of Courtallam $>$ Thirunelveli $>$ Trichy $>$ Coimbatore $>$ Arni (Thirvannamalai) > Padavedu (Thiruvannamalai) > Chengalpattu $>$ Dindigul. Significant variations in the antioxidant activities with respect to the sampling locations were observed. The dynamics of variation in the antioxidant content is possibly associated with the expression of variety of genes during various developmental stages of the plant or because of the environmental factors arising from seasonal variations ${ }^{11-13}$. In general, environmental factors like variations in the altitude, temperature, precipitation, etc varies rapidly. The sampling locations of this study falls between 36 to 1778 m elevations representing diverse climatic conditions, which is associated with antioxidant activity $^{14}$. Literature review also suggests that antioxidant activity is influenced by various species of compounds. This implies that the intake of antioxidant compounds by the G.sylvestre plants will have vital impact on the antioxidant activity of the plant samples collected from diverse locations. The variation of intake depends on the texture of the soil and the seasonal conditions ${ }^{15}$. It is noteworthy that the observed antioxidant activity is much superior to that of the total leaf extract reported recently ${ }^{16}$.
The compounds identified in the crude extracts of G.sylvestre are mostly belongs to terpenes, alcohols, hydrocarbons, alkaloids and its derivatives. From the literature search, these compounds are found to be known for their therapeutic properties and are previously reported in many different medicinal plants. Some of these compounds are separately isolated in extracts and are used as antimicrobial and radical scavenging agents in medicine formulations. This study shows that the chemical compounds isolated in different crude extracts of G.sylvestre could be used as a vital source of antioxidant for food and pharmaceutical industry.

The crude extracts from the G.sylvestre leaves were subjected for GCMS analysis for identifying compounds. Various studies using GCMS has revealed the influence of different solvents in isolating the phytochemical constituents with medicinal values from crude extracts of medicinal plants ${ }^{17}$. The major chemical compounds identified in the G.sylvestre crude extracts such as Inositol, 1-deoxy- found in methanol extract, 2-Pentanone, 3,3,4,4-tetramethyl found in methanol and ethanolic extracts, Tetratriacontane and Hexadecane found in benzene, chloroform, ethyl acetate and hexane extracts, Eicosane form benzene, chloroform and ethyl acetate extracts, Heneicosane found in benzene, ethyl acetate and hexane extracts, Phthalic acid, di(2-propylpentyl) ester found in hexane and methanol extracts, Squalene, Phytol, n-Hexadecanoic acid and Stigmasterol found in all the extracts are chemically or biologically active compounds (Table $8)$.

\section{Table 8: Therapeutic activity of compounds identified in the leaves of G.sylvestre.}

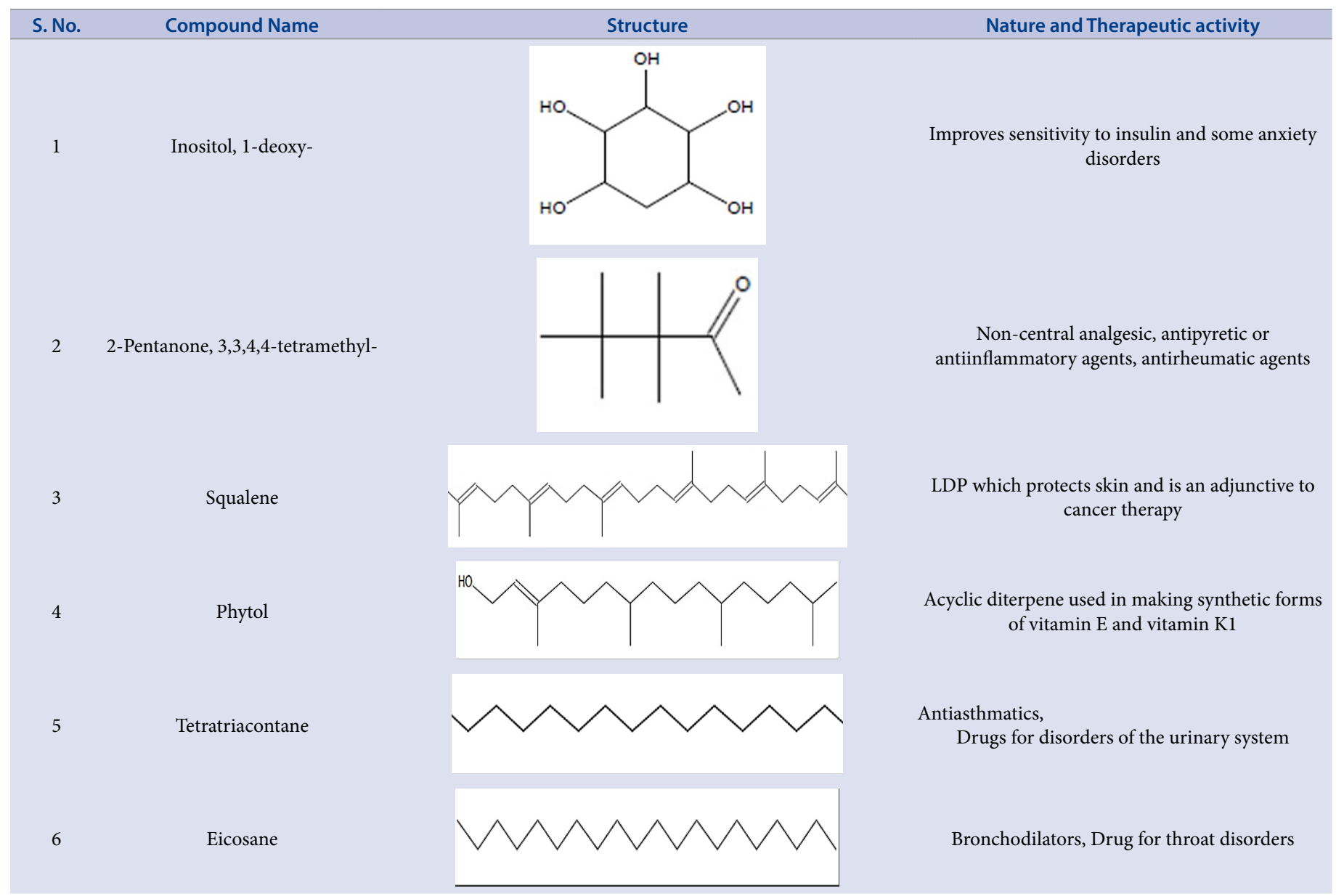




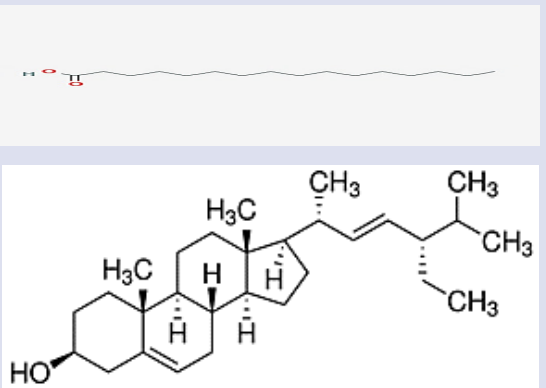

Stigmasterol

8

9

Hexadecane

Phthalic acid, di(2-propylpentyl) ester
prostaglandin-E2 9-reductase) inhibitor

It has a role as a vitamin and a plant metabolite, Bronchodilator

Plant metabolite

Anticancer activity

Antineoplastic, oviposition-attractant pheromone (for trapping mosquitoes)

\section{CONCLUSION}

The present study revealed a number of compounds isolated in different solvents and its efficiency. Also, the effect of locations on the antioxidant activity exhibited by the plant was revealed. The whole plant can be used as a good source of antioxidant. Future research will be taken up for the isolation and characterization of individual compounds from the crude extracts of Gymnema sylvestre and tested for in-vivo studies for further understanding the activities of plant compounds.

\section{ACKNOWLEDGEMENT}

The Authors would like to thank Nanotechnology Research Centre (NRC), SRM IST for providing facilities.

\section{REFERENCES}

1. Yeh GY, Eisenberg DM, Kaptchuk TJ, Phillips RS. Systematic review of herbs and dietary supplements for glycemic control in diabetes. Diabetes Care. 2003;26(4):1277-94.

2. Satdive RK, Abhilash P, Fulzele DP. Antimicrobial activity of Gymnema sylvestre leaf extract. Fitoterapia (Milano). 2003;74(7-8):699-701.

3. Arunachalam KD, Arun LB, Annamalai SK, Arunachalam AM. Potential anticancer properties of bioactive compounds of Gymnema sylvestre and its biofunctionalized silver nanoparticles. International Journal of Nanomedicine. 2015;10:31.

4. Sinsheimer JE, Rao GS, Mcllhenny HM, Smith RV, Maassab HF, Cochran $\mathrm{KW}$. Isolation and antiviral activity of the gymnemic acids. Experientia. 1968;24(3):302-3

5. Subashini MS, Rajendran P. In vitro screening of anti HBV and anti HIV properties of Gymnema sylvestre R. Br leaves from Kolli Hills, Tamilnadu, India. Int J Curr Microbiol Appl Sci. 2015:4:542-7.

6. Rates SM. Plants as source of drugs. Toxicon. 2001;39(5):603-13.
7. Thakur GS, Sharma R, Sanodiya BS, Pandey M, Prasad GB, Bisen PS. Gymnema sylvestre: an alternative therapeutic agent for management of diabetes. Journal of Applied Pharmaceutical Science. 2012;2(12):1-6.

8. Butler MS, Robertson AA, Cooper MA. Natural product and natural product derived drugs in clinical trials. Natural Product Reports. 2014;31(11):1612-61.

9. Blois MS. Antioxidant determinations by the use of a stable free radical. Nature. 1958;181(4617):1199-200

10. Cieśla Ł, Kryszeń J, Stochmal A, OleszekW, Waksmundzka-Hajnos M. Approach to develop a standardized TLC-DPPH test for assessing free radical scavenging properties of selected phenolic compounds. Journal of Pharmaceutical and Biomedical Analysis. 2012;70:126-35.

11. Kumari R, Singh S, Agrawal SB. Effects of supplemental ultraviolet-B radiation on growth and physiology of Acorus calamus L.(sweet flag). Acta Biol Cracoviensia Ser Bot. 2009;51:19-27.

12. Kondakova V, Tsvetkov I, Batchvarova R, Badjakov I, Dzhambazova T, Slavov S. Phenol compounds-qualitative index in small fruits. Biotechnology \& Biotechnological Equipment. 2009;23(4):1444-8.

13. Li HY, Hao ZB, Wang XL, Huang L, Li JP. Antioxidant activities of extracts and fractions from Lysimachia foenum-graecum Hance. Bioresource Technology. 2009;100(2):970-4

14. Sati, P., Pandey, A., Rawat, S. and Rani, A. Phytochemicals and antioxidants in leaf extracts of Ginkgo biloba with reference to location, seasonal variation and solvent system. Journal of Pharmacy Research. 2013;7(9):804-9.

15. Iqbal S, Bhanger MI. Effect of season and production location on antioxidant activity of Moringa oleifera leaves grown in Pakistan. Journal of food Composition and Analysis. 2006;19(6-7):544-51.

16. Naik D, Dandge C, Rupanar S. Chemical Examination and Evaluation of Antioxidant and Antimicrobial Activities of Essential Oil from Gymnema sylvestre. Journal of Essential Oil Research. 2011;23:12-20.

17. Al Hashmi LS, Hossain MA, Weli AM, Al-Riyami Q, Al-Sabahi JN. Gas chromatography-mass spectrometry analysis of different organic crude extracts from the local medicinal plant of Thymus vulgaris L. Asian Pacific Journal of Tropical Biomedicine. 2013;3(1):69. 


\section{GRAPHICAL ABSTRACT}
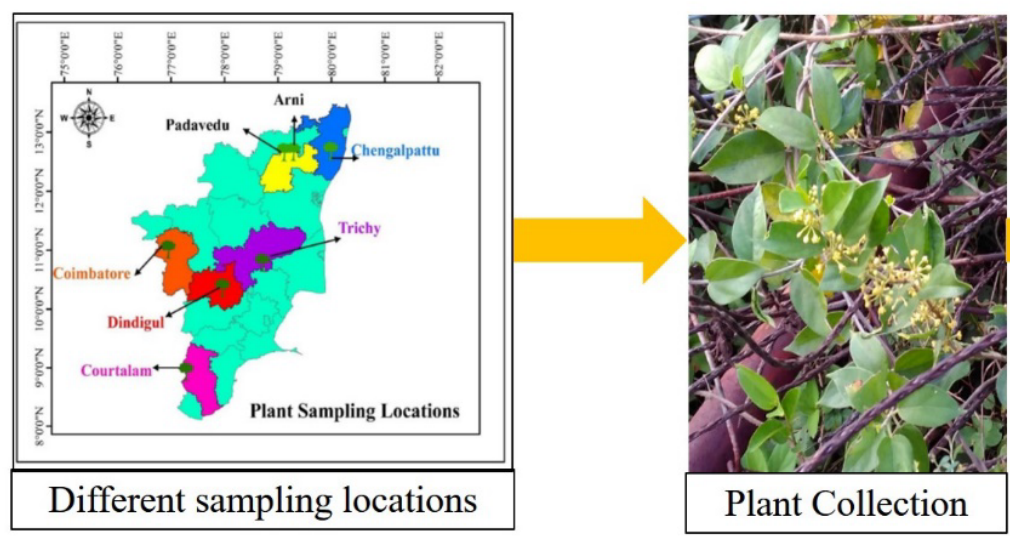

Effect of Sampling:

Antioxidant activity by DPPH radical scavenging
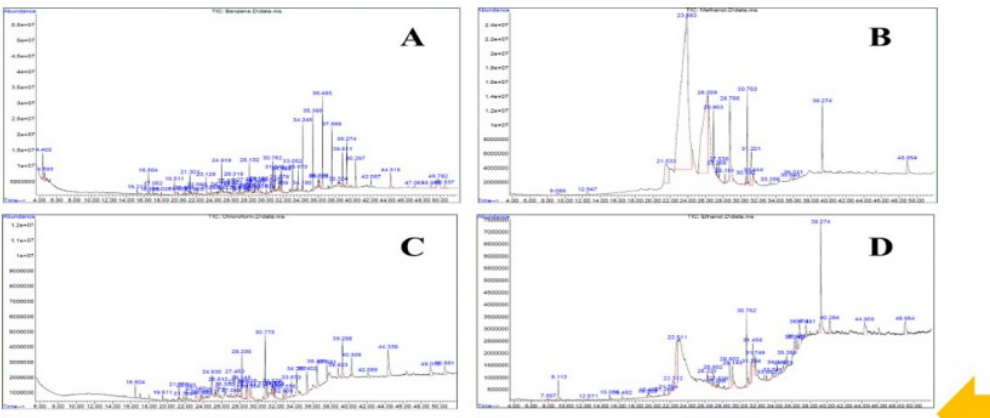

$\mathbf{E}$

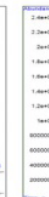

$\mathbf{F}$

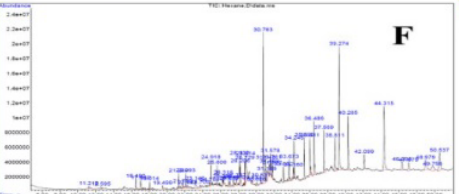

\section{Effect of solvent:}

Estimation of chemical constituents by GCMS

\section{ABOUT AUTHORS}

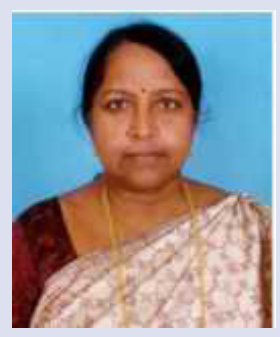

\section{PROF. (MRS.) KANTHA DEIVI ARUNACHALAM}

She is the Dean for the Center for Environmental Nuclear Research, SRM Institute of Science and Technology, Tamil Nadu. She completed her Doctoral degree in Microbial toxicology at Madurai Kamaraj University. She got specialization in Environmental Engineering from Memorial University of Newfoundland, Canada. She has vast teaching and research experience of 40 years both in Canada and India. 13 students under her guidance have been awarded doctoral degrees and the count still goes on. She has attracted research funds from both Government and private sectors and is a possessor of various awards of excellence issued by various Scientific Societies from India and abroad. She has a total of 79 publications with a cumulative impact factor of 114 .

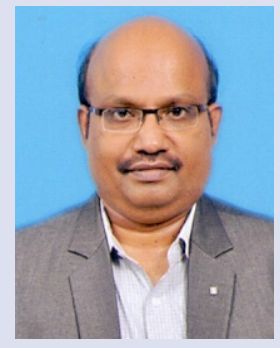

\section{DR. S. SUNDARPANDIAN}

He is a medical doctor with masters specialization in Anatomy. He is working at SRM Medical College and research Centre, Chennai. Currently he is doing his Ph.D degree under the guidance of Prof. Kantha D. Arunachalam from Center for Environmental Nuclear Research, SRM Institute of Science and Technology, Tamil Nadu. With a total of 37 publications in various reputed journals, he also has teaching experience of 26 years and is handling courses for medical college students of various disciplines. He has received various awards from medical societies and for his par excellence in the field of teaching. 


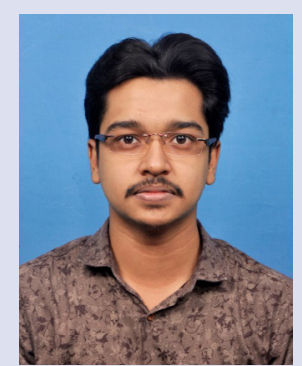

\section{MOHAMMED JUNAID HUSSAIN. D}

$\mathrm{He}$ is a research scholar under the guidance of Prof. Kantha D. Arunachalam at Center for Environmental Nuclear Research, SRM Institute of Science and Technology, Tamil Nadu. He completed his Master's degree in Biotechnology at University of Madras, Chennai. He did internship and masters dissertation project at Department of Biotechnology, CSIR- CLRI under the guidance of Dr. N.R. Kamini. With the core subject of his project being enzymology, he has a technical knowledge in bioprocessing and enzymology and has experience in handling relevant instruments. Currently he is working on mitigation studies for the effects of radiation using plant based bioactive compounds.

\section{SATHISH KUMAR. K}

$\mathrm{He}$ is a research scholar under the guidance of Prof. Kantha D. Arunachalam at Center for Environmental Nuclear Research, SRM Institute of Science and Technology, Tamil Nadu. He completed his Master's degree in Biotechnology at Bharathiyar University, Coimbatore. He has 2 years of work experience in the quality control at Biocon Limited. He is expertise in microbiological practices and his current research focuses on the fabrication of biofunctionalized electrospun nanofiber for wound healing applications.

\section{SARAVANAN. M}

Saravanan $\mathrm{M}$ is a post graduate student from the School of Bioengineering, SRM Institute of Science and Technology, Kattankulathur, Tamil Nadu. He has completed his under graduate in degree in Biotechnology from B. S. Abdur Rahman Crescent Institute of Science and Technology, Vandalur, Tamil Nadu. He is an organized researcher with reputed lab experience inclusive of strong background knowledge in Biotechnology. A highly enthusiastic person who is keen to learn the research advancement in the field of life sciences. He has successfully completed his UG project entitled at IITM Research park where he gained practical skills upon life science industry exposure.

Cite this article: Subramanian S, Dowlath MJH, Karuppannan SK, Saravanan M, Arunachalam KD. Effect of Solvent on the Phytochemical Extraction and GC-MS Analysis of Gymnema sylvestre. Pharmacogn J. 2020;12(4):749-61. 